\title{
FACTORS ASSOCIATED WITH DIMINISHED COUGH INTENSITY IN COMMUNITY-DWELLING ELDERLY USING DAY CARE SERVICES: A PILOT STUDY
}

\author{
R. Mikiya ${ }^{1}$, C. Momoki², D. Habu ${ }^{3}$
}

\begin{abstract}
Purpose: We investigated factors affecting diminished cough intensity in community-dwelling elderly using day care services. Participants and Methods: A total of 61 elderly males and females aged $\geq 65$ years who were certified to receive long-term adult day care services were enrolled in this study. Assessments included: Cough intensity (assessed using cough peak flow measurements, as well as possible determinants of cough intensity, lifestyle, and demographic characteristics), nutritional status (using the Mini Nutritional Assessment-Short Form), dietary intake (using the Dietary Variety Score), routine activity (using the Japanese version of the International Physical Activity Questionnaire), care-related factors (including day care services utilization and an oral exercise regimen) as well as age, need for long-term care, gender, sarcopenia status, the Charlson Comorbidity Index, and body mass, limb skeletal mass, and respiratory indices. Results: A reduced cough peak flow (odds ratio 4.46, 95\% confidence interval: 1.08-18.43) was associated with sarcopenia and was weakly (not significantly) associated with age, gender, and the Mini Nutritional Assessment-Short Form score. Conclusion: A reduced cough peak flow was independently associated with sarcopenia and associated with age, gender, and nutritional status.
\end{abstract}

Key words: Elderly, cough intensity, sarcopenia.

\section{Introduction}

Dysphagia is associated with diminished cough intensity (assessed using cough peak flow [CPF] measurements) and impaired airway clearance. Such individuals are predisposed to aspiration pneumonia (1). A study performed by Bach et al. showed that in patients with a neuromuscular disorder who show $\mathrm{CPF}$ $\leq 270 \mathrm{~L} / \mathrm{min}$, inadequate expectoration of sputum could cause respiratory insufficiency (2). Previous studies have reported that a reduced CPF in elderly is associated with age, lifestyle, and the maximal inspiratory pressure (MIP) $(3,4)$. Kim et al. reported that age-related reduction in routine activity accelerates respiratory muscle weakness (5). Expiratory muscle weakness causes generation of inadequate pleural pressure during coughing, thereby

1. Department of Physical Therapy, Faculty of Health Sciences, Morinomiya University of Medical Sciences, Suminoe-ku, Osaka-shi, Osaka, Japan; 2. Department of Food and Nutrition, Faculty of Contemporary Human Life Science, Tezukayama University, Nara-shi, Nara, Japan ; 3. Department of Medical Nutrition, Graduate School of Life Science, Osaka City University, , Sumiyoshi-ku Osaka-shi, Osaka, Japan

Corresponding Author: Ryosuke Mikiya, Department of Physical Therapy, Faculty of Health Sciences, Morinomiya University of Medical Sciences, 1-2616 Nankokita, Suminoe-ku, Osaka-shi, Osaka 559-8611, Japan, Email: mikiya@ morinomiya-u.ac.jp (06-6616-6911, FAX06-6616-6912) reducing the expiratory flow rate. This diminishes the cough intensity in elderly and can predispose these individuals to respiratory diseases.

Sarcopenia is an age-related reduction in muscle strength associated with reduced activity and loss of skeletal muscle mass. Recently, sarcopenia has gained attention as a major factor causing frailty in the elderly. The reduction in muscle mass in patients with sarcopenia can occur even in the respiratory muscles; thus, elderly with sarcopenia demonstrate decreased respiratory muscle strength and endurance (6). Sarcopenia is primarily an age-related condition; however, disuse atrophy secondary to malnutrition and reduced physical activity and disease-induced cachexia can cause sarcopenia (7-9). A study has reported that the prevalence of sarcopenia in elderly Japanese (aged $\geq 65$ years) is approximately $20 \%$ for both males and females (10). Thus, a significant number of community-dwelling elderly may have sarcopenia and diminished cough intensity. Previous studies $(5,6)$ have reported that sarcopenia may be closely associated with a reduced CPF secondary to respiratory muscle weakness.

Adult day care service (day care) in Japan is a system in which community-dwelling elderly with diminished physical or cognitive function who are certified as needing support or long-term care visit facilities specified 
by ordinances of the Ministry of Health, Labor, and Welfare or day care centers catering to elderly. These facilities provide assistance with bathing, toileting, eating, and other routine activities, as well as functional training to such individuals. An individual must have a formal certification of their need for long-term care in order to avail themselves of the day care services under longterm care insurance. A few studies have identified an association between $\mathrm{CPF}$ and respiratory function in community-dwelling elderly who are certified as needing support or long-term care and who use day care services. However, no studies have examined the multifaceted effects of lifestyle (including physical activity and nutritional status) and baseline physical characteristics (age, gender, and body composition) on CPF. Thus, we investigated the effect of the aforementioned factors on the CPF in elderly who are certified as needing support or long-term care and who avail themselves of day care services.

Figure 1

Study participants flow

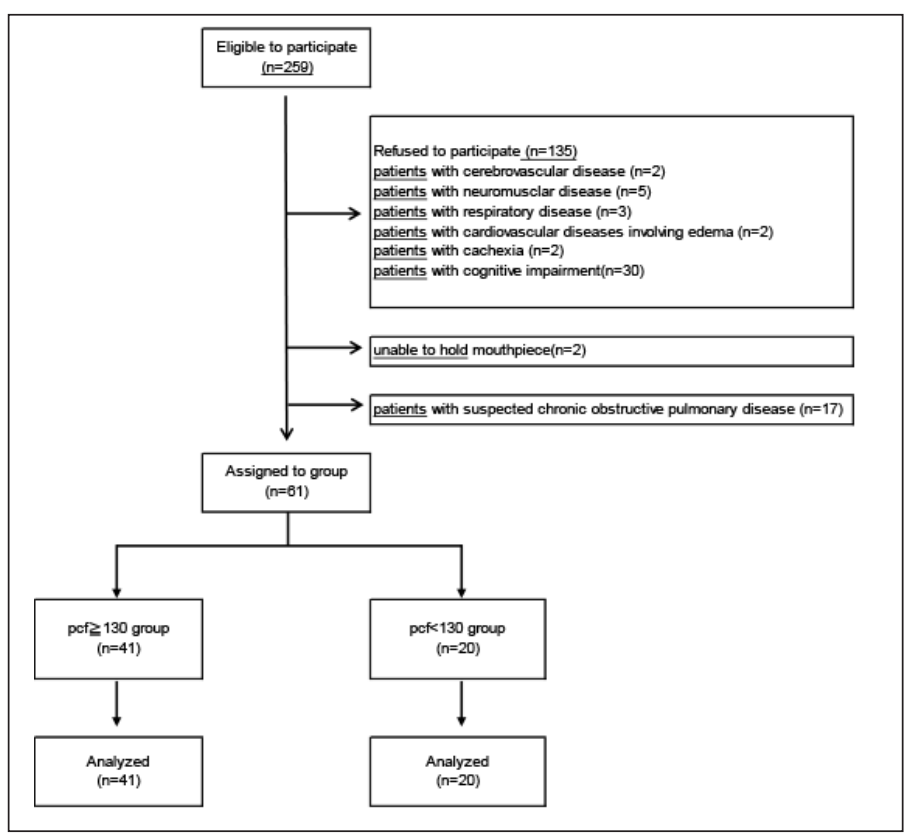

\section{Participants and methods}

This study is a cross-sectional research design. We investigated 259 elderly who were certified as needing support or long-term care and who used day care services across 4 adult day care centers. The purpose of this study was well explained to potential participants prior to enrollment, and 61 elderly aged $\geq 65$ years ( 15 males, 46 females) provided written consent to participate in this study. The mean age of participants was $80.4( \pm 6.2)$ years. Individuals with cerebrovascular, neuromuscular, respiratory, or cardiovascular disorders with pedal edema, those with cancer or any other condition causing cachexia, individuals previously diagnosed with dementia, and those who were unable to appropriately use the mouthpiece of the spirometer were excluded from the study. Additionally, individuals with a forced expiratory volume $<70 \%$ in $1 \mathrm{~s}$ (based on spirometry evaluation) with suspected chronic obstructive pulmonary disease (COPD) were excluded (Fig. 1).

CPF was used as an indicator of voluntary cough intensity. CPF was ranked in tertile, with a flow rate $\geq 130 \mathrm{~L} / \mathrm{min}$ indicating a high CPF and a flow rate $<130 \mathrm{~L} / \mathrm{min}$ indicating a low CPF. CPF was measured with a spirometer (Autospiro AS-507, Minato Medical Science, Co., LTD, Osaka, Japan). CPF evaluation was performed with all participants seated in an upright position. Each participant was instructed to firmly hold the mouthpiece to their lips and cheeks to prevent air from escaping, and a nose clip was used to prevent the escape of air from the nose. After participants had attained their maximal inspiratory level, they were instructed to cough thrice, and the maximum value was used. Additionally, respiratory function indices such as the forced vital capacity (FVC) were assessed. A respiratory dynamometer was connected to the aforementioned device, and the maximal expiratory pressure (MEP) and the MIP were measured thrice, and the maximum value recorded during the 3 attempts was used. A previous study has reported the usefulness of the Mini Nutritional Assessment (MNA) instrument to assess nutritional status, particularly in elderly (11). The MNA is an effective screening tool for nutritional status assessment, and the short form of the MNA (MNA-SF) includes the first 6 items present in the MNA (diminished food intake over the past 3 months, weight loss over the past 3 months, mobility, the presence or absence of psychological problems, body mass index (BMI), and calf circumference). A previous study has reported the usefulness of the MNA-SF (12). In the current study, the MNA-SF was used as a screening tool for nutritional status assessment. Participants were divided into 2 groups-adequately nourished individuals (MNA-SF score $\geq 12$ points) and those at risk of being malnourished (MNA-SF score $\leq 11$ points). The Dietary Variety Score (DVS) was used to assess participants' dietary variety. The DVS assesses the frequency of weekly consumption of 10 types of foods including the following: meat, fish and shellfish, eggs, dairy, soybean products, green and yellow vegetables, seaweed, fruits, potatoes, and foods containing fats and oils (13). A response of "I eat this food almost every day" for a given food group was scored as 1 point and other responses were scored as 0 points. The total score was considered the DVS. Based on a study performed by Kumagai et al, individuals were categorized as those with marked dietary variety (indicated by a DVS score $\geq 4$ points) and those with lesser dietary variety (indicated by a DVS score $\leq 3$ points) (13). The International Physical Activity Questionnaire (IPAQ) is an internationally standardized questionnaire used across 12 countries to assess routine physical activity in 
Table 1

The characteristics of analysis participants

\begin{tabular}{|c|c|c|c|c|}
\hline & & \multicolumn{3}{|c|}{ cough peak flow $(\mathrm{L} / \mathrm{m})$} \\
\hline & & $\geq 130$ & $<130$ & \\
\hline \multicolumn{2}{|l|}{ Variables } & $\mathrm{n}=41$ & $\mathrm{n}=\mathbf{2 0}$ & P value \\
\hline \multicolumn{5}{|c|}{ Basic information } \\
\hline & Age(years) & $79.0(76.0-85.0)$ & $82.5(77.8-86.0)$ & 0,166 \\
\hline \multicolumn{5}{|c|}{ Lifestyle information } \\
\hline & Physical activity(Mets/week) & $528(198-693)$ & $371(198-558.5)$ & 0,384 \\
\hline \multicolumn{5}{|c|}{ Body Composition } \\
\hline & Height(cm) & $156(149.2-163.0)$ & 151(146.8-153.3) & 0,011 \\
\hline & Weight(kg) & $56.0(47.5-61.5)$ & $50.1(46.1-51.8)$ & 0,1 \\
\hline & Body mass index $\left(\mathrm{kg} /{ }^{2}\right)$ & 22.6(19.9-24.5) & 21.7(20.7-23.1) & 0,842 \\
\hline & Skeltal mass index $\left(\mathrm{kg} /{ }^{2}\right)$ & $6.6(5.9-7.0)$ & $5.9(5.4-6.2)$ & 0,002 \\
\hline \multicolumn{5}{|c|}{ Pulmonary Function } \\
\hline & $\mathrm{FVC}(\mathrm{L})$ & $2.07(1.80-2.65)$ & $1.52(1.14-1.68)$ & $<0.001$ \\
\hline & FEV1.0\% & $78.8(76.8-83.2)$ & 83.5(75.9-91.0) & 0,282 \\
\hline & $\mathrm{MIP}(\mathrm{cmH} 2 \mathrm{O})$ & $37.9(26.2-46.3)$ & 21.6(19.9-31.8) & $<0.001$ \\
\hline & $\mathrm{MEP}(\mathrm{cmH} 2 \mathrm{O})$ & $62.3(48.4-78.4)$ & $42.9(34.8-53.1)$ & $<0.001$ \\
\hline
\end{tabular}

The values were described as median interquartile range (IQR), and Mann-Whitney U test was performed. FVC: Forced Vital Capacity, FEV: Forced expiratory volume 1.0(s)\%, MIP: Maximum inspiratory pressure, MEP: Maximum expiratory pressure.

adults aged 18-65 years (14). The Japanese version of the IPAQ was used to assess physical activity. The long and short forms of the IPAQ obtain information regarding the number of days a participant performs intense and moderate physical activity on a weekly basis. The energy expended during the weekly physical activity can be calculated (in Kcal). The Japanese version of the IPAQ short form (IPAQ-SF) was used in the current study to calculate the energy expended during physical activity based solely on the intensity of that activity. Based on a study reported by Murase et al., physical activity can be categorized as moderate level activity with energy expenditure $\geq 600$ metabolic equivalents (METs)/ week and low level activity with energy expenditure $<600$ METs/week (15).

The Asian Working Group for Sarcopenia (AWGS) defines sarcopenia as a condition characterized by loss of muscle strength (grip strength: $<26.0 \mathrm{~kg}$ in males, $<18.0$ $\mathrm{kg}$ in females), diminished limb function (usual gait speed $<0.8 \mathrm{~m} / \mathrm{s}$ ), loss of muscle strength and concomitant diminished limb function, as well as a low limb skeletal muscle mass index (SMI) based on bioelectrical impedance analysis (BIA) $\left(<7.0 \mathrm{~kg} / \mathrm{m}^{2}\right.$ for males, $<5.7$ $\mathrm{kg} / \mathrm{m}^{2}$ for females) (16). Grip strength was measured using a Smedley hand dynamometer (DT-2177, Toei Light, Sōka, Japan). Grip strength of the dominant hand was measured thrice, and the maximum value among the 3 recordings was used. The usual gait speed $(\mathrm{m} / \mathrm{s})$ was calculated based on the time needed to walk $10 \mathrm{~m}$ in a straight line with a build-up and a slow-down. Limb skeletal muscle mass was measured with an electrical impedance plethysmograph (InBody S10, InBody Japan, Inc., Tokyo, Japan) using BIA. In participants with some metallic device in their body, the limb skeletal muscle mass was calculated using Sanada's predictive formula. The result was divided by the square of the height (in $\mathrm{m}^{2}$ ) to calculate the SMI (17). Participants were categorized as those who performed oral exercises (massaging the salivary glands, stretching from the shoulders to the fingers, cheek and tongue movements, and vocal cord exercises) and those who did not perform oral exercises. Other demographic characteristics evaluated included age, gender, need for long-term care, day care service utilization (in h), the Charlson Comorbidity Index (indicating the type and severity of the comorbidity), and BMI. Continuous variables were categorized as binary categorical variables. Age was categorized as $\geq 75$ years and $<75$ years, the level of long-term care needed in participants was categorized as need for $\geq$ level 2 longterm care or the need for level 1 long-term care/support, and the use of day care services (in h) was categorized as prolonged use of $7 \mathrm{~h}$ or brief use of $\leq 3 \mathrm{~h}$. The Charlson Comorbidity Index $w$ as $\geq 1$ point or 0 points $=$ no comorbidity (18). Similar to the protocol followed by the National Health and Nutrition Examination Survey, elderly aged $\geq 65$ years were categorized as those 
Table 2

Univariate and multivariate OR and 95\% CI for cough peak flow

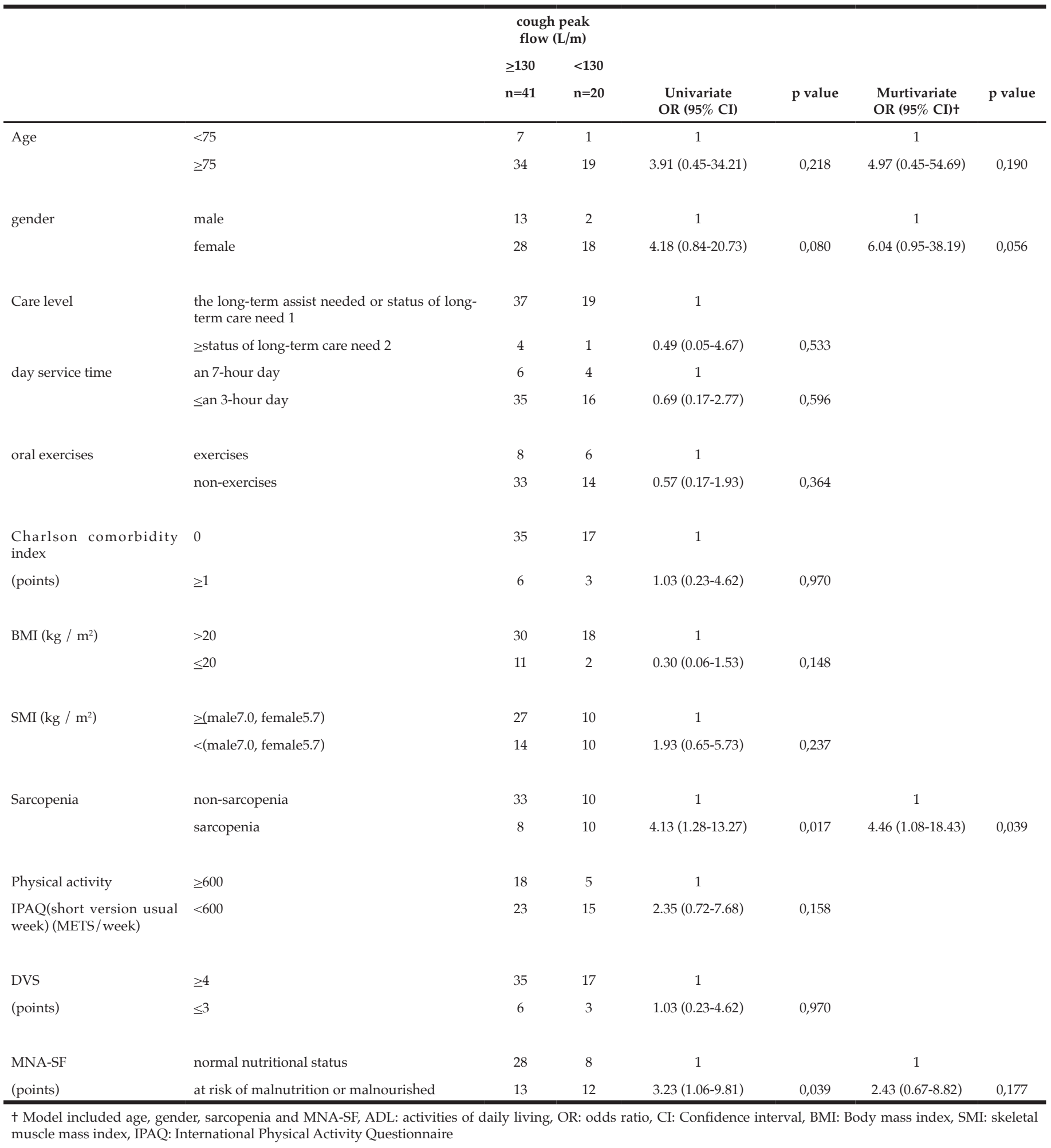

with BMI $\leq 20 \mathrm{~kg} / \mathrm{m}^{2}$ (indicating that the individual is underweight) and those with BMI $>20 \mathrm{~kg} / \mathrm{m}^{2}$ (19). Based on the AWGS definition, males were categorized as those with SMI $\geq 7.0 \mathrm{~kg} / \mathrm{m}^{2}$ and those with $\mathrm{SMI}<7.0 \mathrm{~kg} / \mathrm{m}^{2}$. Females were categorized as those with $\mathrm{SMI} \geq 5.7 \mathrm{~kg} / \mathrm{m}^{2}$ and those with SMI $<5.7 \mathrm{~kg} / \mathrm{m}^{2}$.

The CPF was ranked in tertiles for statistical analysis. A Mann-Whitney $U$ test was used for an intergroup comparison (those with a CPF $\geq 130 \mathrm{~L} / \mathrm{min}$ and those with a CPF $<130 \mathrm{~L} / \mathrm{min}$ ) of participants' quantitative 
variables related to demographic characteristics. Univariate analysis was performed with CPF $\geq 130 \mathrm{~L} / \mathrm{min}$ and $\mathrm{CPF}<130 \mathrm{~L} / \mathrm{min}$ as the outcomes. Subsequently, we performed multiple logistic regression analysis to analyze 4 factors without multicollinearity and with a high odds ratio (age, gender, sarcopenia status, and the MNA-SF score) in univariate analysis. All statistical analyses were performed using SAS, version 9.4 (SAS Institute Inc., Cary, NC USA), and a $p$ value $<0.05$ was considered statistically significant. This study was approved by the Ethical Review Board of Morinomiya University (approval no.: 2016-097).

\section{Results}

Demographic characteristics of the participants in this study are shown in Table 1 . Comparison between 41 participants with a high CPF $(\geq 130 \mathrm{~L} / \mathrm{m})$ and 20 participants with a low CPF $(<130 \mathrm{~L} / \mathrm{m})$ showed significant statistical differences in height, SMI, and respiratory indices (FVC, MIP, and MEP). All these parameters were higher in participants with a high CPF. As a result of univariate and multivariate regression analyses, reduced CPF as the response variable and individual factors as independent variables indicated that sarcopenia (odds ratio [OR] 4.46, 95\% confidence interval [CI] 1.08-18.43) was independently associated with a reduced CPF. Additionally, a reduced CPF was weakly associated with age (OR 4.97, 95\% CI 0.45-54.69), gender (OR 6.04, 95\% CI 0.95-38.19), and the MNA-SF score (OR $2.43,95 \%$ CI 0.67-8.82) (Table 2).

\section{Discussion}

The results of our study revealed that sarcopenia was independently and significantly associated with a reduced CPF in elderly who used day care services and needed support or long-term care. Additionally, sarcopenia was weakly associated with MNA-SF score ( $\leq 11$ points was malnutrition), as well as with age and gender.

Sarcopenia is typically an age-related condition, although it can occur secondary to diminished activity, malnutrition, or cachexia (7-9). Sarcopenia can affect the respiratory muscles with consequent reduction in respiratory muscle strength and endurance (6). Diminished respiratory muscle strength secondary to sarcopenia was significantly associated with a reduced CPF in our current study. A recent study indicated that the capacity of the pectoralis major observed on chest computed tomography may serve as a predictor of aspiration pneumonia (20). A previous study has reported that sarcopenia is associated with the aspiration pneumonia-related mortality rate in elderly patients. Another study has reported that sarcopenia is a risk factor for postoperative respiratory complications (21, 22). Our current study investigated the factors associated with aspiration pneumonia in elderly who needed support or long-term care, and our results suggested that sarcopenia may contribute to the mechanism of diminished cough intensity.

An association (albeit weak) between CPF and MNASF score could be explained by a presumed association between malnutrition and diminished muscle strength. Malnutrition reduces respiratory muscle strength and maximal voluntary ventilation. Arora et al. reported that improved nutrition reduces the occurrence of pulmonary disease (23). Malnutrition reduces respiratory muscle mass, and the lack of energy reduces respiratory muscle activity. Age was also weakly associated with a reduced CPF. A previous study has reported that expiratory and inspiratory muscle strength decrease with age (24), and a similar trend was noted in our current study. Another previous study indicated that respiratory muscle strength is greater in males than in females (6), and this gender difference presumably explains the reduced CPF. Notably, this gender difference was evident even when male and female participants having the same physique were investigated. However, the exact cause that explains this difference remains unclear.

Comparison of participants with a high CPF $(\geq 130$ $\mathrm{L} / \mathrm{m})$ and participants with a low CPF $(<130 \mathrm{~L} / \mathrm{m})$ revealed significant differences in respiratory indicesthese indices were higher in participants with a high $\mathrm{CPF}$. This finding concurs with a previous study, which reported that $\mathrm{CPF}$ was associated with respiratory indices (25). Significant differences between two groups were observed in height and SMI in that these parameters were higher in participants with a high CPF; however, no significant differences were observed in BMI between the 2 groups. This finding could be attributed to the fact that a few participants were diagnosed with obesity and sarcopenia, i.e., they demonstrated a large amount of fat and sarcopenia but a short stature. We intend to investigate this issue in future studies.

A limitation of this study was its small sample size. We preliminarily investigated $\mathrm{CPF}$ in a limited sample, and the statistical power of the study was limited. However, sarcopenia was significantly and independently associated with a reduced $\mathrm{CPF}$, which suggests that sarcopenia is a relatively potent contributor to a reduced $\mathrm{CPF}$. Nonetheless, other potent factors cannot be ruled out. We intend to perform large-scale studies in future to clarify the association between a reduced CPF and the aforementioned individual factors.

As stated, we intend to study the aforementioned association followed by a longitudinal evaluation of day care services. If a cause-and-effect relationship can be identified, it would help in determining the factors that require intervention to prevent reduction in $\mathrm{CPF}$ and consequent aspiration pneumonia. This finding could lead to new findings with regard to community efforts to obviate the need for long-term care.

In conclusion, this study reveals that sarcopenia is a potent risk factor for diminished cough intensity, 
which is known to predispose patients to aspiration pneumonia. In our view, our current findings are highly significant in that they provide specific quantitative data highlighting the association between sarcopenia and the development of aspiration pneumonia via the mechanism of "diminished cough intensity." Additionally, a reduced $\mathrm{CPF}$ was observed to be weakly associated with nutritional status by MNA-SF, age, and gender. A reduced $\mathrm{CPF}$ is significantly associated with sarcopenia.

\section{Funding and Conflict of interest: None to disclose.}

Ethical standard: This study was approved by the Ethical Review Board of Morinomiya University of Medical Sciences (approval no: 2016-097). Data were only collected from those repondants who signed informed consent. This study was conducted in accordance with the Declaration of Helsinki.

\section{References}

1. Marik PE, Kaplan D: Aspiration pneumonia and dysphagia in the elderly. Chest, 2003, 124: 328-336.

2. Bach JR, Ishikawa Y, Kim H: Prevention of pulmonary morbidity for patients with Duchenne muscular dystrophy. Chest, 1997, 112: 1024-1028.

3. Freitas FS, Ibiapina CC, Alvim CG, et al.: Relationship between cough strength and functional level in elderly. Rev Bras Fisioter, 2010, 14: 470-476.

4. Bahat G, Tufan A, Ozkaya H, et al.: Relation between hand grip strength, respiratory muscle strength and spirometric measures in male nursing home residents. Aging Male, 2014, 17: 136-140.

5. Kim J, Davenport P, Sapienza C: Effect of expiratory muscle strength training on elderly cough function. Arch Gerontol Geriatr, 2009, 48: 361-366.

6. Chen HI, Kuo CS: Relationship between respiratory muscle function and age, gender, and other factors. J Appl Physiol, 1989, 66: 943-948.

7. Delmonico MJ, Harris TB, Lee JS, et al.: Alternative definitions of sarcopenia, lower extremity performance, and functional impairment with aging in older men and women. J Am Geriatr Soc, 2007, 55: 769-774.

8. Goodpaster BH, Park SW, Harris TB, et al.: The loss of skeletal muscle strength, mass, and quality in older adults: The health, aging and body composition study. J Gerontol A Biol Sci Med Sci, 2006, 61: 1059-1064.

9. Cruz-Jentoft AJ, Baeyens JP, Bauer JM, et al.: Sarcopenia: European consensus on definition and diagnosis: Report of the European Working Group on Sarcopenia in Older People. Age Ageing, 2010, 39: 412-423.

10. Yamada M, Nishiguchi S, Fukutani N, et al.: Prevalence of sarcopenia in community-dwelling Japanese older adults. J Am Med Dir Assoc, 2013, 14 911-915.

11. Izawa S, Kuzuya M, Okada K, et al.: The nutritional status of frail elderly with care needs according to the mini-nutritional assessment. Clin Nutr, 2006, 25: 962-967.

12. Rubenstein LZ, Harker JO, Salvà $\mathrm{A}$, et al.: Screening for malnutrition in geriatric practice: Developing the Short-form Mini Nutritional Assessment (MNA-SF). J Gerontol A Biol Sci Med Sci, 2001, 56: 366-372.

13. Kumagai $\mathrm{S}$, Watanabe $\mathrm{S}$, Shibata $\mathrm{H}$, et al.: Effects of dietary variety on declines in high-level functional capacity in elderly people living in a community. Nihon Koshu Eisei Zasshi, 2003, 50: 1117-1124. In Japanese.

14. Craig CL, Marshall AL, Sjöström M, et al.: International physical activity questionnaire: 12-country reliability and validity. Med Sci Sports Exerc, 2003, 35: 1381-1395.

15. Murase N, Katsumura T, Ueda C, et al.: Validity and reliability of Japanese version of the International Physical Activity Questionnaire. J Health Welfare Stat, 2002, 49: 1-9. In Japanese.

16. Chen LK, Liu LK, Woo J, et al. Sarcopenia in Asia: Consensus report of the Asian Working Group for Sarcopenia. J Am Med Dir Assoc, 2014, 15: 95-101.

17. Sanada K, Miyachi M, Yamamoto K, et al.: Prediction models of sarcopenia in Japanese adult men and women. Jpn J Phys Fitness Sport, 2010, 59: 291-302.

18. Charlson ME, Pompei P, Ales KL, et al.: A new method of classifying prognostic comorbidity in longitudinal studies: development and validation. J Chronic Dis, 1987, 40: 373-383.

19 National Health and Nutrition Survey, Retrieved September 30, 2018, from https:// www.mhlw.go.jp/content/10904750/000351576.pdf

20. Sakaguchi K, Hara S: Capacity of the pectoralis major muscle may be a prognostic factor for aspiration pneumonia. Adv Aging Res, 2017, 6: 101-117.

21. Maeda K, Akagi J: Muscle mass loss is a potential predictor of 90 -day mortality in older adults with aspiration pneumonia. J Am Geriatr Soc, 2017, 65: e18-e22.

22. Soma D, Kawamura YI, Yamashita S, et al.: Sarcopenia, the depletion of muscle mass, an independent predictor of respiratory complications after oncological esophagectomy. Dis Esophagus, 2018.

23. Arora NS, Rochester DF. Respiratory muscle strength and maximal voluntary ventilation in undernourished patients. Am Rev Respir Dis, 1982, 126: 5-8.

24. Enright PL, Kronmal RA, Manolio TA, et al.: Respiratory muscle strength in the elderly. Correlates and reference values. Cardiovascular Health Study Research Group. Am J Respir Crit Care Med, 1994, 149: 430-438.

25. Kang SW, Bach JR. Maximum insufflation capacity: vital capacity and cough flows in neuromuscular disease. Am J Phys Med Rehabil, 2000, 79: 222-227. 